BULLETIN OF THE

AMERICAN MATHEMATICAL SOCIETY

Volume 79, Number 1, January 1973

\title{
CURVATURE MEASURES FOR PIECEWISE LINEAR MANIFOLDS ${ }^{1}$
}

\author{
BY F. J. FLAHERTY
}

Communicated by S. S. Chern, May 30, 1972

Let $K$ be a convex cell of dimension $m$ in Euclidean $n$-space, $R^{n}$. The volume of the tubular neighborhood of radius $\rho$ around $K$ is given by a polynomial, in $\rho$,

$$
\sum_{p} \sum_{i} H^{p}\left(K_{p}^{i}\right) \frac{v_{n-m}}{v_{m-p}} \frac{\rho^{n-p}}{n-p} \int_{c_{p}^{i}} d S^{m-p-1}
$$

where $H^{p}$ is the $p$-dimensional Hausdorff measure in $R^{n}, d S^{k}$ is the volume element of the standard unit sphere in $R^{k}, v_{k}$ is $H^{k-1}\left(S^{k-1}\right), K_{p}^{i}$ is a face of dimension $p, c_{p}^{i}$ is the outer normal angle determined by $K_{p}^{i}, p$ varies from 0 to $m, i$ varies from 1 to $N_{p}=$ the number of faces of dimension $p$, and $m<n$.

From this formula we can define the pth curvature measure of $K$ as follows. For any bounded Borel set $A \subset R^{n}$,

$$
\sigma_{p}(A)=\sum_{i} H^{p}\left(A \cap K_{p}^{i}\right) \frac{1}{v_{m-p}} \int_{c_{p}^{i}} d S^{m-p-1} .
$$

In addition to being measures, the $\sigma_{p}$ are invariant under the full Euclidean group of rigid motions in $R^{n}$ and satisfy the following strong stability property.

THEOREM 1. Let $L$ be a $k$-dimensional affine subspace of $R^{n}$ and $\xi(n, k)$ the volume element of the manifold $E(n, k)$ of all $k$-dimensional affine subspaces in $R^{n}$. Then

$$
\int_{L \cap K \neq \varnothing} \sigma_{j}(L \cap K) \xi(n, k)=c_{j} \sigma_{n-k+j}(K),
$$

where $c_{j}$ is a constant depending on $n, m, k$.

Given a piecewise linear manifold $K$ of dimension $m$, with boundary $\partial K$, piecewise linearly embedded in $R^{n}$ one can also define the $p$ th curvature measure of $K$. For any bounded Borel set $A \subset R^{n}$,

AMS (MOS) subject classifications (1970). Primary 53C65, 49F20; Secondary 57C35.

${ }^{1}$ Research supported by the Sonderforschungsbereich at the University of Bonn. 


$$
\sigma_{p}(A)=\sum H^{p}\left(A \cap S_{p}\right) \frac{1}{v_{m-p}} \int_{\bar{s}_{p}} d S^{m-p-1}
$$

where the summation is over all cells $s_{p}$ in the $p$-skeleton of $K$ and where $\bar{s}_{p}=\sum_{k} \sum_{j}(-1)^{k} \bar{c}_{k, j}$ with $k$ running from 0 to $p$ and $j$ running over all interior angles in a convex decomposition of the star of $s_{p}$; moreover the $\bar{s}_{p}$ are chains on $S^{m-p-1}$.

Note that the supports of the $\sigma_{p}$ are contained in $\partial K$ for $p \leqq m-1=$ $n-1$ since $K$ is a manifold, and that the curvature measures are now Radon measures (in the sense of Bourbaki).

THEOREM 2. The curvature measures $\sigma_{p}$ of a piecewise linear submanifold $K$ of $R^{n}$ are invariant under the group of rigid motions of $R^{n}$ and are stable in the sense of Theorem 1.

A Radon measure $\phi$ on $R^{n}$ is called a geometric measure of dimension $m$ on $R^{n}$ iff $\phi$ is a real linear combination of Radon measures $\phi_{j}, j=$ $0,1, \ldots, m$ for which the following conditions hold: $\phi_{j}(A)=0$ if the dimension of $A$ is less than $j, \phi_{j}$ is rigid motion invariant, $\phi_{0}$ is a topological invariant and

$$
\int_{L \cap K \neq \varnothing} \phi_{j}(K \cap L) \xi(n, k)=b_{j} \phi_{n-k+j}(K) \quad(n-j \leqq m),
$$

where the notation is the same as in Theorem $1, K$ a piecewise linear submanifold of $R^{n}$ and $b_{j}$ is a constant depending only on dimensional considerations.

THEOREM 3. Let $\phi$ be a geometric measure of dimension $m$ on $R^{n}$, then there exist real numbers $a_{i}$ such that

$$
\phi=\sum_{i} a_{i} \sigma_{i}, \quad i=0,1, \ldots, m,
$$

in the sense that, for any piecewise linear submanifold $K$ of $R^{n}$,

$$
\phi(K)=\sum_{i} a_{i} \sigma_{i}(K) .
$$

Blaschke has proved the curvature measures of simplicial complexes generate the space of finitely additive rigid motion invariant set functions $\phi$ that are bounded and for which $\phi_{3}$ is $S L(3, R)$ invariant [1]. Hadwiger has proved that the curvature measures of convex sets generate the space of finitely additive rigid motion invariant set functions that are continuous with respect to Hausdorff distance [4]. Our definition of the curvature measures extends these measures to sets that may not have positive reach in the sense of Federer [3]. The curvature measures can, of course, be 
defined in the smooth case [2] and together with the techniques given here, the curvature measures may also be defined for piecewise differentiable manifolds.

\section{BIBLIOGRAPHY}

1. W. Blaschke, Vorlesungen über Integralgeometrie, Zweites Heft, Teubner, Leipzig und Berlin, 1937.

2. S. S. Chern, On the kinematic formula in integral geometry, J. Math. Mech. 16 (1966), 101-118. MR 33 \#6564.

3. H. Federer, Curvature measures, Trans. Amer. Math. Soc. 93 (1959), 418-491. MR 22 \#961.

4. H. Hadwiger, Vorlesungen über Inhalt, Oberfläche und Isoperimetrie, Springer-Verlag, Berlin, 1957. MR 21 \# 1561.

Mathematics Institute, University of Bonn, 53 Bonn, West Germany

Department of Mathematics, Oregon State University, Corvallis, Oregon 97331 (Current address.) 